\title{
Phase space properties and chaotic transport for a particle moving in a time dependent step potential well
}

\author{
Diogo Ricardo da Costa ${ }^{\mathrm{a}, \mathrm{b}, *}$, Iberê L. Caldas ${ }^{\mathrm{a}}$, Edson D. Leonel ${ }^{\mathrm{c}}$ \\ ${ }^{a}$ Instituto de Física da USP, Rua do Matão, Travessa R, 187, Cidade Universitária, 05314-970 São Paulo, SP, Brazil \\ ${ }^{\mathrm{b}}$ School of Mathematics, University of Bristol, Bristol BS8 1TW, United Kingdom \\ ${ }^{\mathrm{c}}$ Departamento de Física, UNESP, Univ Estadual Paulista, Av.24A, 1515, Bela Vista, 13506-900 Rio Claro, SP, Brazil
}

\section{A R T I C L E I N F O}

\section{Keywords:}

Chaos

Step potential well

Chaotic transport

Phase space properties

\begin{abstract}
A B S T R A C T
Some dynamical properties for an ensemble of non-interacting classical particles along chaotic orbits and transport properties over the chaotic sea for the problem of a step and time dependent potential well are considered. The dynamics of each particle is described by a two-dimensional, nonlinear and area preserving mapping for the variables energy and time. The phase space is of mixed-type and contains periodic islands, a set of invariant KAM curves and chaotic seas. The chaotic orbits are characterized by the use of Lyapunov exponents. Transport over the chaotic sea is considered and scaling exponents are obtained. A sticky region around a chain of periodic islands produces local and temporarily trapping of the dynamics and discussions of the rearrangement of the phase space are made.
\end{abstract}

(c) 2014 Elsevier Inc. All rights reserved.

\section{Introduction}

The investigation of problems described by potential wells or barriers where the particles are subject to the effect of noise, or the barriers are assumed to move in time is a subject considered by many researchers from the last decades [1-5]. Applications in the scientific community may consider either classical and quantum cases. To illustrate applications of the subject in Bose-Einstein condensates, recently effects of spin-orbit coupling were considered to describe the tunneling from wells in a Bose-Einstein condensate [6], quantum phase transition induced by atom-pair tunneling [7] and selftrapping appearing as a consequence of nonlinear effects [8]. Applications of the double well system may also be observed in resonators where cooling is possible due to a control of the optical forces leading the system to the ground state [9] or experiments in an optomechanical system where a cavity mode is coupled with a membrane in a double well potential [10]. In classical description, recent results discussing tunneling from a time dependent potential well using the transfer-matrix technique and shown that the time dependence affects the transmission probability of electrons in the potential [11], the formalism of Heun confluent was used to solve a family of quasi-exactly double well [12]. Moreover analytical expressions for the probability of finding a particle in the problem of a Brownian particle in the problem of a double well was recently obtained [13] while the Fokker-Planck formalism was used to obtain the transmission probability from a time dependent potential well considering either fast, slow and with small and large noise [14]. It was also considered the description of the survival probability and escape from one well to the other one due to noise [15]. Indeed the survival probability was

\footnotetext{
* Corresponding author at: Instituto de Física da USP, Rua do Matão, Travessa R, 187, Cidade Universitária, 05314-970 São Paulo, SP, Brazil. E-mail addresses: diogo_cost@hotmail.com, diogocost2@gmail.com (D.R. da Costa).
} 
shown to be exponential when the escape from one well to the other one is quick while long trapping leads the survival probability to change from exponential to a power law. The slower decay is marked mainly by orbits that survive long in the dynamics. In mixed phase space, they are related to sticky regions near periodic regions. During the dynamics the particle passes near enough a regular region and stays trapped there for a while when it then leaves such region.

The model we consider in this paper consists of a classical particle which is confined inside a potential box which contains two periodically time-dependent square wells. Our main goal is therefore to understand and describe some dynamical as well as transport properties along the phase space, particularly in the chaotic region, focusing on the escaping dynamics. The Hamiltonian that describes the model is of the type $H(x, p, t)=p^{2} /(2 m)+V(x, t)$ where $x, p$ and $t$ correspond to the position, momentum coordinates and time respectively. As we will see in the next section, the potential $V(x, t)$ is controlled by different control parameters. If they are changed accordingly, a phase transition from integrability - characterized by a constant energy of the particle - to non-integrability - where the particle may be observed either in a chaotic or regular dynamics - is observed. The dynamics of the model is described by a two-dimensional non-linear area preserving mapping, for the variables energy and time. The phase space of the model is of mixed type and shows periodic islands surrounded by a chaotic sea which is characterized by a positive Lyapunov exponent. The size of the chaotic sea depends on the control parameters and is limited by a set of invariant KAM curves, which prevents the unlimited energy growth of the particle. Average properties of the chaotic sea can be described using scaling approach. Near the phase transition from integrability to non-integrability, critical exponents characterize the laws which describe how the average properties of the chaotic sea [16], like average energy of the particle or the deviation of the average energy, behave near such a criticality. The critical exponents may be used to define or set the transition into classes of universality.

When a hole is introduced in the energy axis, the histogram of frequency for the transport of particles, which we observe to be scaling invariant, grows rapidly until it reaches a maximum and then decreases towards zero at sufficiently long times. Generally the behavior of the survival probability of the particles in the dynamics as a function of time is observed to be exponential for short times, reaching a crossover time and turning to a slower-decay regime, due to sticky regions observed in the phase space [3]. It happens because the model has a mixed phase space which leads to non-uniformity and sticky domains that produce anomalous transport [17-19]. A sticky region traps a particle in the phase space and the escape from this region happens at a very long time after the entrance. These sticky regions can be identified by the use of Lyapunov exponents, which have typical behavior in such regions [20]. The changeover from fast exponential decay to a slower decay was also observed recently [15] where the slower decay was due to orbits trapped in metastable regime.

This paper is organized as follows. In Section 2 we describe the model and the mapping that characterizes the dynamics of the particle. The numerical results and discussions are also presented in this section. Final discussions are drawn in Section 3.

\section{The model, the map and numerical results}

In this section we construct the equations that describe the dynamics of the model. Some dynamical properties for chaotic orbits along the phase space are discussed by the use of scaling arguments. The model consists of a classical particle of mass $m$ confined inside a box of infinite potential at the borders which contains a time varying step potential. Allusions of the time dependent potential can be made as corresponding to the potential created by atoms placed in sequence along an infinite long and symmetric chain while the oscillations may denote phonon effects or either the contact of the chain with a thermal bath. It means that the potential well is getting energy from a thermal bath and is transferring it to the particle. In our approach, we assume this transference is periodic in time leading to a mixed phase space structure. If the transference is stochastic, a random diffusion in energy should indeed be observed therefore producing an unlimited growth. A sketch of the potential is shown in Fig. 1.

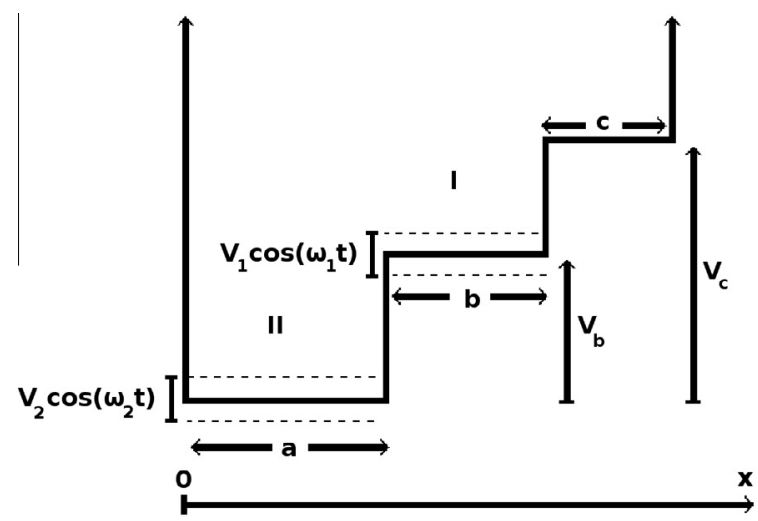

Fig. 1. Sketch of the time-dependent potential. 
We emphasise that different kinds of potential shape lead to similar dynamics. For a chain of infinitely many and symmetric oscillating square wells with their bottoms moving periodically and synchronised in time, the dynamics leads to diffusion in space, as we can see in the Fig. 2(a). Due to the symmetry of this problem, it is possible to consider only one symetric potential with periodic boundary conditions, as shown in Fig. 2(b). Moreover it is possible to see that this last figure is also symetric with respect to the middle of the figure, as shown in Fig. 1.

In this paper we consider the potential as shown in Fig. 1, and it is given by

$$
V(x, t)=\left\{\begin{array}{ll}
\infty, & \text { if } x \leqslant 0 \text { or } x \geqslant(a+b+c) \\
V_{2} \cos \left(\omega_{2} t\right), & \text { if } 0<x<a \\
V_{b}+V_{1} \cos \left(\omega_{1} t\right), & \text { if } a \leqslant x<(a+b) \\
V_{c}, & \text { if }(a+b) \leqslant x<(a+b+c)
\end{array},\right.
$$

where the control parameters $a, b, c, V_{b}, V_{c}, V_{1}, V_{2}, \omega_{1}, \omega_{2}$ are constants.

The dynamics of the model is made via a discrete mapping which updates the variables energy and time at $x=a$ when the particle enters at region II. Starting with an initial energy $E \leqslant V_{b}$ the full description of the dynamics is made by the consideration of three different situations: (i) the particle does not have enough energy to escape region II and is reflected left hand side; (ii) the particle escapes region II but does not have enough energy to escape region I and is reflected backwards and; (iii) the particle escapes region I travels the distance $c$ until reflects left hand side with the same energy and enters regions I and II. Let us start with case (i) first. We assume that the particle is at the position $x=a$ moving left hand side with an initial energy $E=E_{n} \geqslant V_{b}$ at the time $t=t_{n}$. When the particle enters region II it suffers a sudden change in its kinetic energy $K_{n}^{\prime}=E_{n}-V_{2} \cos \left(\omega_{2} t_{n}\right)$ where $K_{n}^{\prime}=m v_{n}^{\prime 2} / 2$. Given there are no gradients of the potential, the velocity of the particle is constant inside region II and is equal to $\left|v_{n}^{\prime}\right|=\sqrt{2 K_{n}^{\prime} / m}$. The time the particle spends traveling the distance $a$ is equal to $\Delta t_{n}^{\prime}=a /\left|v_{n}^{\prime}\right|$. It then reaches the infinite potential at $x=0$, suffers a reflection and moves backwards until reaches $x=a$. In such a position, the total energy of the particle is equal to $E_{n}^{\prime}=K_{n}^{\prime}+V_{2} \cos \left[\omega_{2}\left(t_{n}+2 \Delta t_{n}^{\prime}\right)\right]$. If $E_{n}^{\prime}<V_{b}+V_{1} \cos \left[\omega_{1}\left(t_{n}+2 \Delta t_{n}^{\prime}\right)\right]$ the particle does not have enough energy to escape region II and suffers a reflection therefore moving left hand side until $x=0$. The escape condition from region II is given by

$$
E_{n}^{\prime}>V_{b}+V_{1} \cos \left[\omega_{1}\left(t_{n}+2 i \Delta t_{n}^{\prime}\right)\right]
$$

where $E_{n}^{\prime}=K_{n}^{\prime}+V_{2} \cos \left[\omega_{2}\left(t_{n}+2 i \Delta t_{n}^{\prime}\right)\right]$ and $i$ is the smallest integer number that makes Eq. (2) true. The probability of observing one successive reflection is larger than observing two, that is larger than observing three and so on. A histogram of frequency for the successive reflections leads to a power law decay with exponent -3 , as shown in Fig. 3.

The exponent -3 was also observed for the traversal time in the problem of a periodically time varying barrier [21]. The exponent -3 however does not seem to be universal. Recently it was observed for a periodically corrugated waveguide [22] that the histogram of successive (multiple) reflections is described by a power law with exponent -3.76 . The periodically corrugated waveguide model consists in a set of two mirrors where one of them is flat and the other is periodically corrugated one. Inside of them there is a beam of light which is specular reflected. The successive reflections are characterized by the reflections that the beam suffers without leaving the corrugated region. Despite the non-linearity of the two models is given or by a sine or cosine functions, which have infinite number of continuous derivatives, the chances of a particle getting trapped in multiple reflections are larger in the potential well than in the corrugated waveguide.

The mapping $T_{A}$ that describes the dynamics of the particle is given by

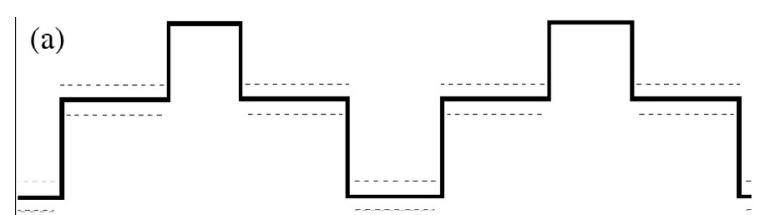

(b)

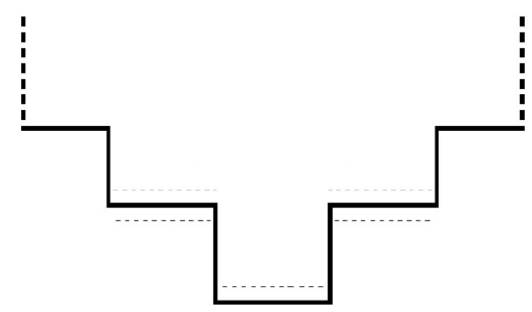

Fig. 2. (a) Sketch of an infinite chain of potential wells; (b) Due to the symmetry of the chain in (a), it is possible to consider only one symmetric potential. 


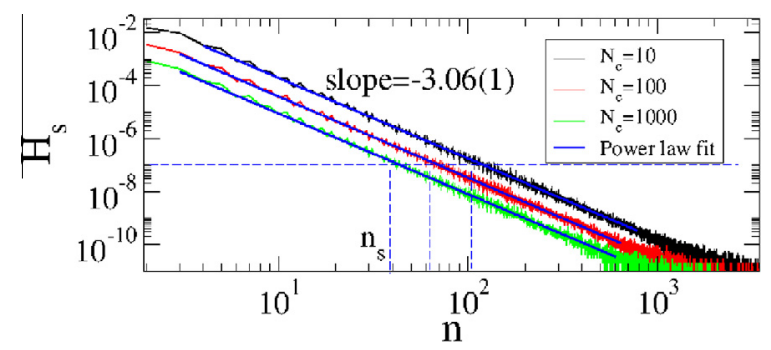

Fig. 3. Plot of the histogram of successive reflections. The parameters used were: $r_{1}=r_{2}=1, \delta_{r}=0.5, \delta_{1}=0.35, \delta_{2}=0.1$ and $\omega_{r}=1$. The orbits were iterated $10^{11}$ times.

$$
T_{A}:\left\{\begin{array}{l}
E_{n}^{\prime}=E_{n}+V_{2}\left[\cos \left(\omega_{2} \Delta t_{f}^{\prime}\right)-\cos \left(\omega_{2} t_{n}\right)\right] \\
\Delta t_{f}^{\prime}=t_{n}+2 i \Delta t_{n}^{\prime}
\end{array} .\right.
$$

When the mapping $T_{A}$ is iterated, the particle enters region I suffering a change in its kinetic energy $K_{n}^{\prime \prime}=E_{n}^{\prime}-$ $\left[V_{b}+V_{1} \cos \left(\omega_{1} \Delta t_{f}^{\prime}\right)\right]$. The velocity of the particle in region I is also a constant and is given by $\left|v_{n}^{\prime \prime}\right|=\sqrt{2 K_{n}^{\prime \prime} / m}$. The time for the particle to travel the distance $b$ is $\Delta t_{n}^{\prime \prime}=b /\left|v_{n}^{\prime \prime}\right|$. The total energy at $x=a+b$ is equal to $E_{n}^{\prime \prime}=V_{b}+$ $K_{n}^{\prime \prime}+V_{1} \cos \left[\omega_{1}\left(\Delta t_{f}^{\prime}+j \Delta t_{n}^{\prime \prime}\right)\right]$ with $j=1$. The mapping $T_{B}$ is written as

$$
T_{B}:\left\{\begin{array}{l}
E_{n}^{\prime \prime}=E_{n}^{\prime}+V_{1}\left[\cos \left(\omega_{1} \Delta t_{f}^{\prime \prime}\right)-\cos \left(\omega_{1} \Delta t_{f}^{\prime}\right)\right] \\
\Delta t_{f}^{\prime \prime}=\Delta t_{f}^{\prime}+j \Delta t_{n}^{\prime \prime}
\end{array}\right.
$$

Reaching the position $x=a+b$ two conditions may occur: (ii) $E_{n}^{\prime \prime} \leqslant V_{c}$, then the particle does not have enough energy to escape from region I and is reflected backwards. From this situation, $j$ must be made $j=2$ and mapping $T_{B}$ is updated. The particle reaches the position $x=a$ and the energy is given by $E_{n+1}=E_{n}^{\prime \prime}$ and $t_{n+1}=\Delta t_{f}^{\prime \prime}$. If the particle escapes region I then case (iii) applies where $\left(E_{n}^{\prime \prime}>V_{c}\right)$. The kinetic energy changes abruptly to $K_{n}^{\prime \prime \prime}=E_{n}^{\prime \prime}-V_{c}$. The velocity is equal to $\left|v_{n}^{\prime \prime \prime}\right|=\sqrt{2 K_{n}^{\prime \prime \prime} / m}$ and the time to move the distance $c$ is equal to $\Delta t_{n}^{\prime \prime \prime}=c /\left|v_{n}^{\prime \prime \prime}\right|$. The particle reaches the infinite potential at $x=a+b+c$, suffers a reflection backwards and travels the distance $c$ again. At the position $x=a+b$ it suffers another change in the kinetic energy $K_{n}^{i v}=E_{n}^{\prime \prime \prime}-\left\{V_{b}+V_{1} \cos \left[\omega_{1}\left(\Delta t_{f}^{\prime \prime}+2 \Delta t_{n}^{\prime \prime \prime}\right)\right]\right\}$. The particle reaches $x=a$ and the final energy is given by $E_{n+1}=K_{n}^{i v}+V_{b}+V_{1} \cos \left[\omega_{1}\left(\Delta t_{f}^{\prime \prime}+2 \Delta t_{n}^{\prime \prime \prime}+\Delta t_{n}^{i v}\right)\right]$, where $\Delta t_{n}^{i v}=b /\left|v_{n}^{i v}\right|$ is the time the particle spends to travel the distance $b$ and with velocity $v_{n}^{i v}=\sqrt{2 K_{n}^{i v} / m}$. The mapping $T_{B}^{\prime}$ is written as

$$
T_{B}^{\prime}:\left\{\begin{aligned}
E_{n+1}= & E_{n}^{\prime \prime}+V_{1} \cos \left(\omega_{1} t_{n+1}\right) \\
& -V_{1} \cos \left[\omega_{1}\left(\Delta t_{f}^{\prime \prime}+2 \Delta t_{n}^{\prime \prime \prime}\right)\right] . \\
t_{n+1}= & \Delta t_{f}^{\prime \prime}+2 \Delta t_{n}^{\prime \prime \prime}+\Delta t_{n}^{i v}
\end{aligned}\right.
$$

As the mapping is constructed, one sees that there are many control parameters and not all of them are relevant for the dynamics. They can be reduced using dimensionless variables therefore we have: $e_{n}=E_{n} / V_{c}, e_{n}^{\prime}=E_{n}^{\prime} / V_{c}, e_{n}^{\prime \prime}=E_{n}^{\prime \prime} / V_{c}$, $e_{n+1}=E_{n+1} / V_{c}, \Delta \phi_{f}^{\prime}=\omega_{1} \Delta t_{f}^{\prime}, \Delta \phi_{f}^{\prime \prime}=\omega_{1} \Delta t_{f}^{\prime \prime}, \phi_{n+1}=\omega_{1} t_{n+1}, \Delta \phi^{\prime}=\omega_{1} \Delta t_{n}^{\prime}, \Delta \phi^{\prime \prime}=\omega_{1} \Delta t_{n}^{\prime \prime}, \Delta \phi^{\prime \prime \prime}=\omega_{1} \Delta t_{n}^{\prime \prime \prime}, \Delta \phi^{i v}=\omega_{1} \Delta t_{n}^{i v}$. The control parameters are written as $\delta_{2}=V_{2} / V_{c}, \delta_{1}=V_{1} / V_{c}, \omega_{r}=\omega_{2} / \omega_{1}, \delta_{r}=V_{b} / V_{c}, r_{1}=b / a, r_{2}=c / a$ and $N_{c}=\omega_{1} a \sqrt{m / 2 V_{c}}$. The parameter $N_{c}$ indeed denotes the number of oscillations that the potential well I makes in the interval of time a particle travels the distance $a$ with total energy $E=V_{c}$. All energies are therefore rescaled as a function of $V_{c}$. It is important to note that the parameters $\delta_{1}$ and $\delta_{2}$ are the dimensionless and rescaled potential. The integrable case is observed for $\delta_{1}=\delta_{2}=0$. On the other hand if $b$ is null, the region I does not exist. If $c$ is null, the static part of the potential disappear. Another possibility arises if $a$ is large. For this case, the particle spends much time in region II, making the bottom of the potential well oscillate many times, increasing the randomness of the system when a particle reaches the position $x=a$. The parameter $\omega_{r}=\omega_{2} / \omega_{1}$ defines an important relationship between $\omega_{1}$ and $\omega_{2}$. For example, if $\omega_{1}=\omega_{2}$ the two potential oscillate in phase and synchronized. If $\omega_{2}=2 \omega_{1}$, the potential well in region II oscillates twice for each oscillation of the potential in the region I, for $\omega_{2}=3 \omega_{1}$, it oscillates three times and so on. If $\omega_{r}$ is irrational, there is no correspondence between the oscillations of these two potential wells leading them to be out of phase.

With this new set of dimensionless variables and considering the following constrains $\delta_{r}+\delta_{1}<1, \delta_{r}-\delta_{1}>\delta_{2}$ and $e_{n}>\delta_{r}+\delta_{1} \cos \left(\phi_{n}\right)$, the mapping $T_{A}$ is rewritten as

$$
T_{A}:\left\{\begin{array}{l}
e_{n}^{\prime}=e_{n}+\delta_{2}\left[\cos \left(\omega_{\mathrm{r}} \Delta \phi_{f}^{\prime}\right)-\cos \left(\omega_{r} \phi_{n}\right)\right] \\
\Delta \phi_{f}^{\prime}=\left[\phi_{n}+2 i \Delta \phi^{\prime}\right] \quad \bmod 2 \pi .
\end{array}\right.
$$

If $e_{n}^{\prime}>\delta_{r}+\delta_{1} \cos \left(\Delta \phi_{f}^{\prime}\right)$, mapping $T_{B}$ applies with $j=1$, then the mapping is written as 


$$
T_{B}:\left\{\begin{array}{l}
e_{n}^{\prime \prime}=e_{n}^{\prime}+\delta_{1}\left[\cos \left(\Delta \phi_{f}^{\prime \prime}\right)-\cos \left(\Delta \phi_{f}^{\prime}\right)\right] \\
\Delta \phi_{f}^{\prime \prime}=\left[\Delta \phi_{f}^{\prime}+j \Delta \phi^{\prime \prime}\right] \quad \bmod 2 \pi
\end{array}\right.
$$

If $e_{n}^{\prime \prime} \leqslant 1$ then $j=2$ and mapping $T_{B}$ is recalculated. Moreover we can make $e_{n+1}=e_{n}^{\prime \prime}$ and $\phi_{n+1}=\Delta \phi_{f}^{\prime \prime}$. On the other hand if $e^{\prime \prime}>1$, then mapping $T_{B}^{\prime}$ is iterated where

$$
T_{B}^{\prime}:\left\{\begin{array}{l}
e_{n+1}=e_{n}^{\prime \prime}+\delta_{1}\left[\cos \left(\phi_{n+1}\right)-\cos \left(\Delta \phi_{f}^{\prime \prime}+2 \Delta \phi^{\prime \prime \prime}\right)\right] \\
\phi_{n+1}=\left[\Delta \phi_{f}^{\prime \prime}+2 \Delta \phi^{\prime \prime \prime}+\Delta \phi^{i v}\right] \quad \bmod 2 \pi .
\end{array}\right.
$$

The auxiliary variables are given by

$$
\begin{aligned}
\Delta \phi^{\prime} & =\frac{N_{c}}{\sqrt{e_{n}-\delta_{2} \cos \left(\omega_{r} \phi_{n}\right)}}, \\
\Delta \phi^{\prime \prime} & =\frac{N_{c} r_{1}}{\sqrt{e_{n}^{\prime}-\delta_{r}-\delta_{1} \cos \left(\Delta \phi_{f}^{\prime}\right)}}, \\
\Delta \phi^{\prime \prime \prime} & =\frac{N_{c} r_{2}}{\sqrt{e_{n}^{\prime \prime}-1}}, \\
\Delta \phi^{i v} & =\frac{N_{c} r_{1}}{\sqrt{e_{n}^{\prime \prime}-\delta_{r}-\delta_{1} \cos \left(\Delta \phi_{f}^{\prime \prime}+2 \Delta \phi^{\prime \prime \prime}\right)}} .
\end{aligned}
$$

The system is integrable for $\delta_{1}=\delta_{2}=0$ and non integrable for any of the possible combinations: (i) $\delta_{1} \neq 0$ and $\delta_{2}=0$; (ii) $\delta_{1}=0$ and $\delta_{2} \neq 0$; or (iii) either $\delta_{1} \neq \delta_{2} \neq 0$. The parameter $N_{c}$ denotes the number of oscillations that the potential well I makes in the interval of time a particle travels the distance $a$ with total energy $E=V_{c}$. Therefore an increase in $N_{c}$ produces more oscillations of the bottom of the moving potential leading to a possible larger exchange of energy of the particle with the potential itself, therefore producing an increase of the chaoticity of the system. Given the mapping is now defined, let us discuss the behavior of the successive reflections. If we defined a characteristic probability in the histogram, say $10^{-7}$, and obtain the number of successive reflections at that probability for different parameters $N_{c}$, a typical decay in power law is observed, as shown in Fig. 4. A power law fitting furnishes a slope of $-0.206(3)$. This behavior can be used to overlap all curves of successive reflections onto a single plot, after a suitable rescaling of the axis, as shown in Fig. 4(b).

The phase space that characterizes the dynamics is shown in Fig. 5 for the control parameters $N_{c}=10, r_{1}=r_{2}=1$, $\delta_{r}=0.5, \delta_{1}=\delta_{2}=0.2$ and $\omega_{r}=1$. The structure observed in the figure shows a set of periodic islands, chaotic seas and invariant KAM curves therefore characterizing the phase space as mixed. Indeed the existence of invariant KAM curves limit the size of the low energy chaotic sea and prevent the particle to diffuse unlimited in the energy. The existence of the

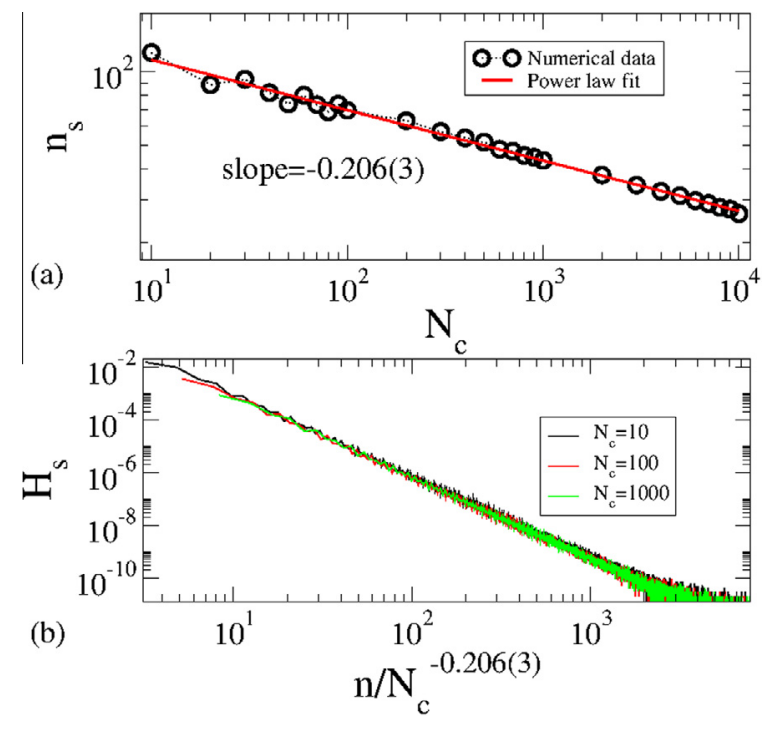

Fig. 4. Plot of the histogram of successive reflections for different control parameters $N_{c}$. Their overlap onto a single plot after a suitable rescaling of the axis. The parameters used were: $r_{1}=r_{2}=1, \delta_{r}=0.5, \delta_{1}=0.35, \delta_{2}=0.1$ and $\omega_{r}=1$. The orbits were iterated $10^{11}$ times. 


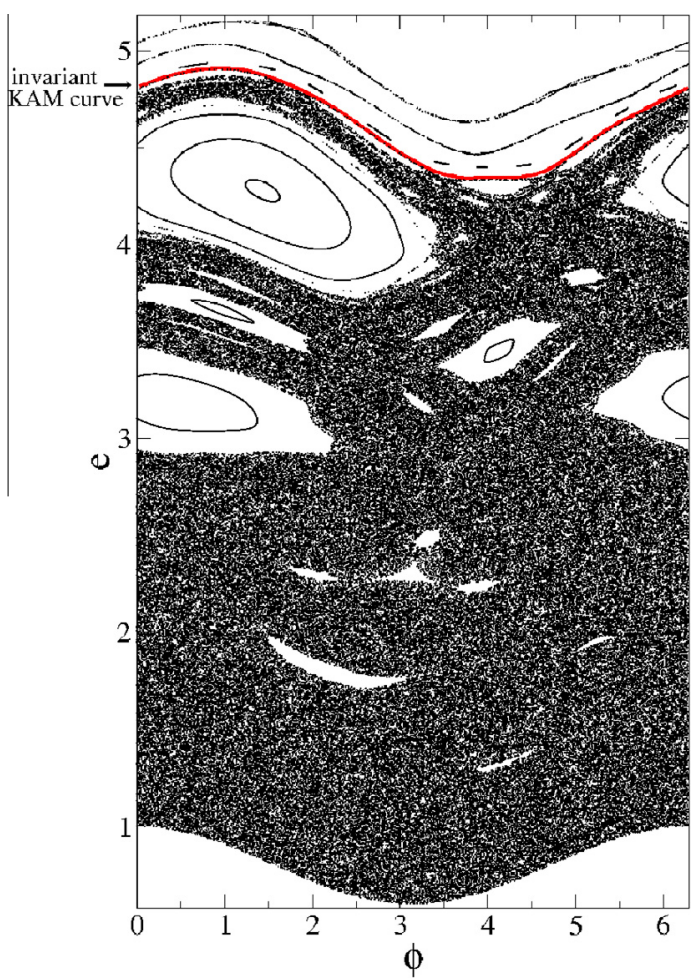

Fig. 5. Plot of a typical phase space. The parameters used were $N_{c}=10, r_{1}=r_{2}=1, \delta_{r}=0.5, \delta_{1}=\delta_{2}=0.2$ and $\omega_{r}=1$. The red dotted line denotes the position of the first invariant KAM curve. (For interpretation of the references to color in this figure legend, the reader is referred to the web version of this article.)

invariant KAM curves leads one to describe some average properties of the chaotic sea using power laws and curves play a fundamental rule in a scaling observed along the chaotic sea as a function of the control parameters. Because of the area preservation property, a particle moving along the chaotic sea can not penetrate KAM island nor a particle in a KAM island can not escape from the island.

Let us now discuss some properties of the phase space. To start with, we discuss the Lyapunov exponents. They are indeed known as an important tool to be used in the characterization of chaotic dynamics. The procedure to obtain the Lyapunov exponents consists in verify if two sufficiently close initial trajectories diverge exponentially fast from each other as time evolves. If the system exhibits at least one positive Lyapunov exponent, then it has chaotic components. The Lyapunov exponents are obtained as [23]

$$
\lambda_{j}=\lim _{n \rightarrow \infty} \frac{1}{n} \ln \left|\Lambda_{j}^{(n)}\right|, \quad j=1,2,
$$

where $\Lambda_{j}^{(n)}$ are the eigenvalues of the matrix $B=\prod_{i}^{n} J_{i}\left(e_{i}, \phi_{i}\right)$ and $J_{i}$ is the Jacobian matrix of the system.

Fig. 6(a) shows a plot of the Lyapunov exponent as a function of $n$ for four different initial conditions in the region of low energy. One sees they fluctuate at the beginning and converge to a regime of constant plateau for large $n$. Fig. 6(b) shows a plot of $\bar{\lambda} \times N_{c}$. One observes a tendency of growth of $\bar{\lambda}$ as $N_{c}$ increases. Fig. 6(c) shows a plot of $\bar{\lambda} \times \omega_{r}$. One notes that $\bar{\lambda}$ is about 1.8 for $\omega_{r}=1$, decays slightly reaching a constant plateau of $\bar{\lambda} \approx 1.4$ for $\omega_{r} \cong 10$, passing by a minimum of $\bar{\lambda} \approx 1.1$ at $\omega_{r} \cong 20$ and approaching the plateau of $\bar{\lambda} \approx 1.4$ for $\omega_{r} \cong 100$. The error bars correspond to the standard deviation of an ensemble of 6 different initial conditions chosen in the chaotic region.

The chaotic sea in the phase space for this model is always limited by a set of invariant KAM curves. They indeed prevent the particle to diffuse unlimited in the phase space. Having this in mind, let us discuss the behavior of the average energy and hence the deviation around the average energy. They are defined as

$$
\bar{e}\left(n, \delta, N_{c}, r\right)=\frac{1}{n} \sum_{i=1}^{n} e_{i}
$$

and the deviation of the average energy as

$$
\rho\left(n, \delta, N_{c}, r\right)=\frac{1}{M} \sum_{j=1}^{M} \sqrt{\overline{e_{j}^{2}}\left(n, \delta, N_{c}, r\right)-{\overline{e_{j}}}^{2}\left(n, \delta, N_{c}, r\right)},
$$



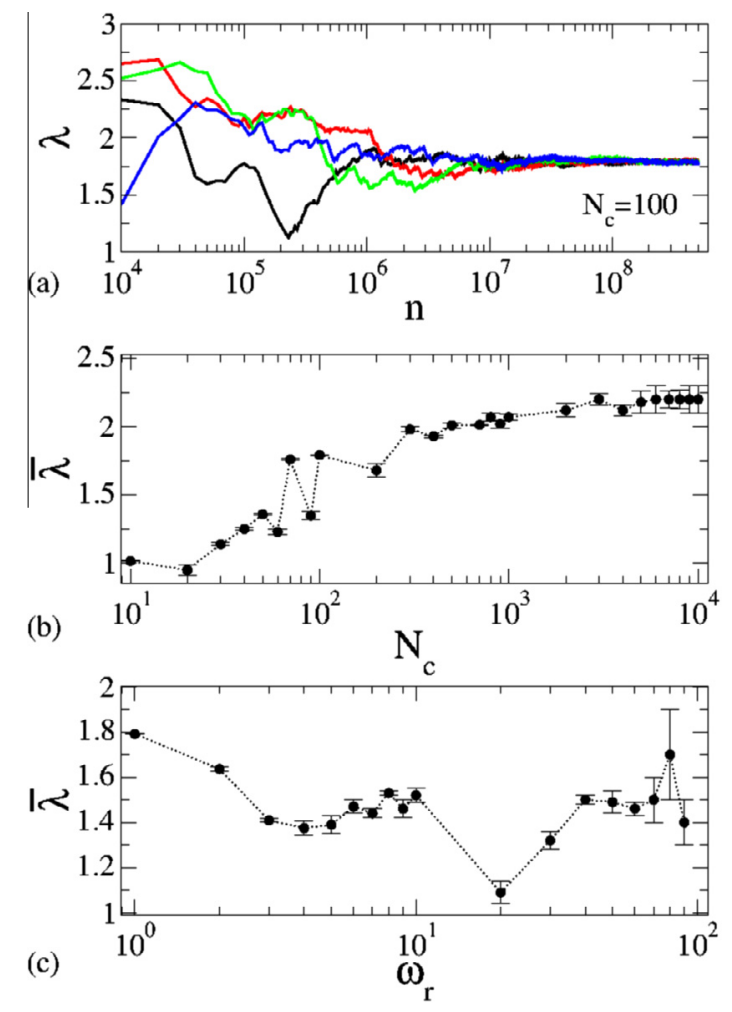

Fig. 6. Plot of: (a) $\lambda \times n$ for 4 different initial conditions chosen along the chaotic sea using $r_{1}=r_{2}=1, \delta_{r}=0.5, \delta_{1}=\delta 2=0.2, N_{c}=100$ and $\omega_{r}=1 ;(b)$ $\bar{\lambda} \times N_{c}$ for $N_{c} \in\left[10^{1}, 10^{4}\right]$ using $\omega_{r}=1 ;$ (c) $\bar{\lambda} \times \omega_{r}$ for $\omega_{r} \in[1,90]$ using $N_{c}=100$.

where $M$ denotes an ensemble of different initial conditions. Indeed we set an initial energy $e_{0}=1.01$ and consider $\phi_{0} \in(0,2 \pi]$ as the different initial phases. Fig. 7 shows a plot of $\rho \times n$ for $N_{c}=5, r_{1}=r_{2}=1, \delta_{r}=0.5, \delta_{1}=\delta_{2}=0.2$ and $\omega_{r}=1$. We see that $\rho$ grows as a power law for small $n$ with slope $\cong 0.5$ and, after a crossover number $n_{x}, \rho$ tends to a regime of saturation $\rho_{\text {sat }}$. A variation of the parameters $N_{c}$ and $\omega_{r}$ produce similar plots but with different crossovers and different saturation. Therefore we propose that

1. For $n \ll n_{x}$, the behavior of $\rho$ can be described as

$$
\rho\left(n, N_{c}, \omega_{r}\right) \propto n^{\beta},
$$

where $\beta \cong 0.5$ is the acceleration exponent.

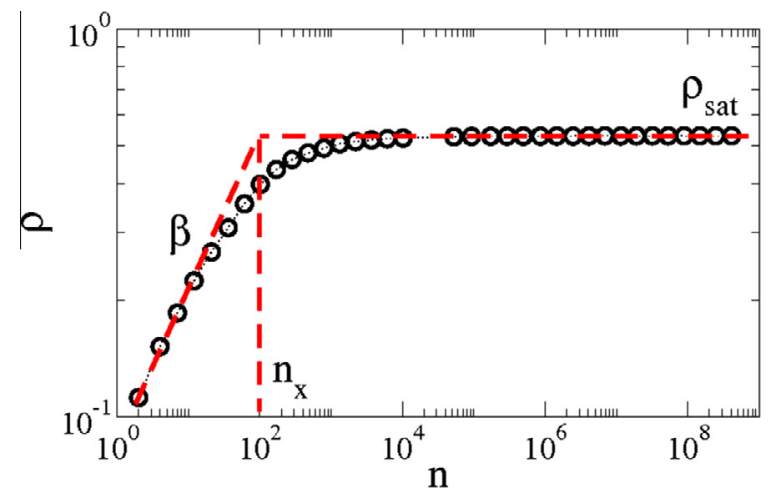

Fig. 7. Plot of $\rho \times n$ for $r_{1}=r_{2}=1, \delta_{r}=0.5, \delta_{1}=\delta_{2}=0.2, \omega_{r}=1$ and $N_{c}=5$. 
2. For $n \gg n_{x}, \rho_{\text {sat }}$ is given by

$$
\rho_{\text {sat }}\left(n, N_{c}, \omega_{r}\right) \propto N_{c}^{\alpha_{1}} \omega_{r}^{\alpha_{2}},
$$

where $\alpha_{1}$ and $\alpha_{2}$ are critical exponents.

3. The characteristic crossover $n_{x}$ is written as

$$
n_{x}\left(n, N_{c}, \omega_{r}\right) \propto N_{c}^{z_{1}} \omega_{r}^{z_{2}},
$$

where $z_{1}$ and $z_{2}$ are dynamical exponents.

The critical exponents can be obtained if the behavior of $\rho_{\text {sat }}$ and $n_{x}$ are obtained as a function of the control parameters. The critical exponents obtained as a function of $N_{c}$ are shown in Fig. 8(a) and (b) while for the control parameter $\omega_{r}$ are shown in Fig. 8(c) and (d). Fitting the curves shown in Fig. 8 by power laws, we obtain that $\alpha_{1}=0.6333(5)$, $\alpha_{2}=0.639(8), z_{1}=1.30(4)$ and $z_{2}=1.31(3)$. The set of exponents obtained can be used to overlap different curves of $\rho$ into a single plot as shown in Fig. 9(a) and (b), after a suitable rescale of the axis.

The behavior of $\rho_{\text {sat }}$ is strongly influenced by the position of the lowest energy invariant KAM curve which defines the laws of the observables in the chaotic sea [24]. The curve is characterized by a typical value of energy $e^{*}$ which fluctuates slightly. Fig. 10(a) shows a plot of the energy of the particle at the invariant KAM curve, which we denote as $e^{*}$, for two different control parameters, as labeled in the figure. Fig. 10(b) and (c) show plots of $e^{*}$ as a function of the control parameters $N_{c}$ and $\omega_{r}$. After fitting a power law in both plots we obtain that the slope is given by $\alpha_{1}=0.654(4)$ and $\alpha_{2}=0.66(1)$. These values are numerically close to those obtained from scaling approach and confirm the influences of the position of the lowest energy invariant KAM curve over observables in the chaotic sea, as discussed in Ref. [24].

Let us now concentrate to investigate some transport properties along the chaotic sea. We consider an ensemble of initial conditions started with low energy, say $e_{0}=2$, and evolve them in time seeking for the number of iterations $n$ that each particle spends until reaches a certain position at the energy axis on the phase space, say $h$. The ensemble is produced by the variation of the initial phase $\phi \in(0,2 \pi]$. When the particle reaches the condition $e_{n+1} \geqslant h$ the number of iterations $n$ is collected, the orbit is stopped and a new initial condition is started. The simulations were carried out using $r_{1}=r_{2}=1, \delta_{r}=0.5$ and $\delta_{1}=\delta_{2}=0.2$. In principle the position $h$ may be either below or above the periodic islands. If below, no regions of trap-
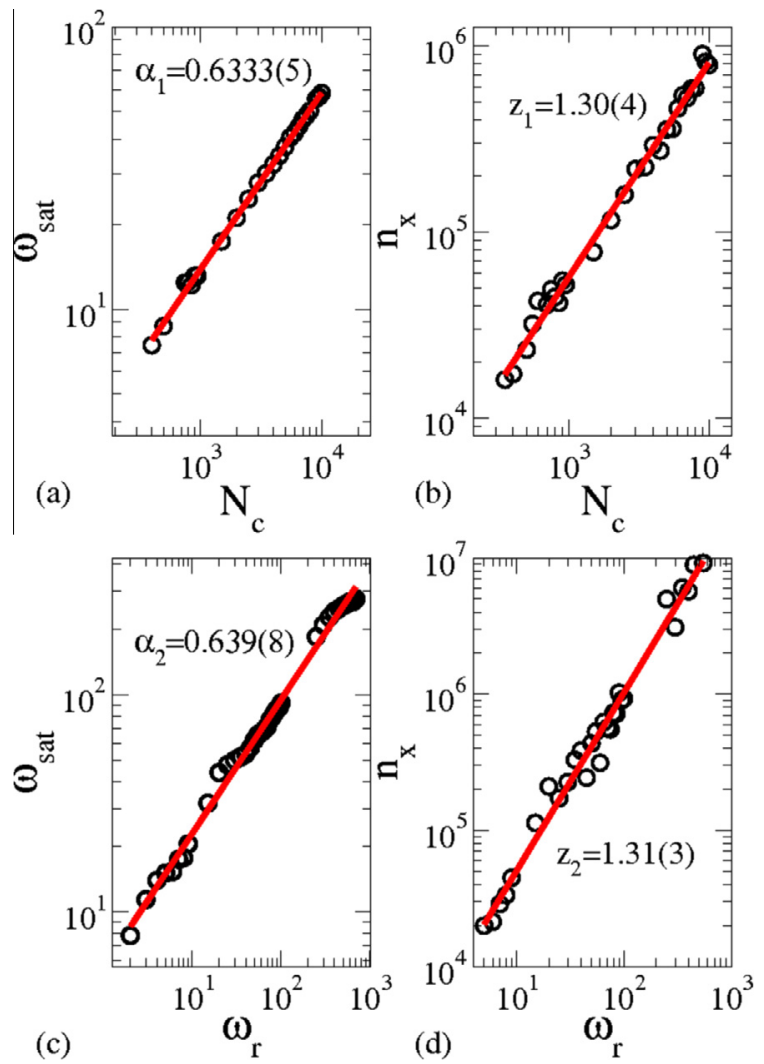

Fig. 8. Plot of: (a) $\rho_{\text {sat }} \times N_{c}$ using $\omega_{r}=1$; (b) $n_{x} \times N_{c}$ using $\omega_{r}=1$; (c) $\rho_{\text {sat }} \times \omega_{r}$ using $N_{c}=100$; (d) $n_{x} \times \omega_{r}$ using $N_{c}=100$. The parameters used were $r_{1}=r_{2}=1, \delta_{r}=0.5$ and $\delta_{1}=\delta_{2}=0.2$ 

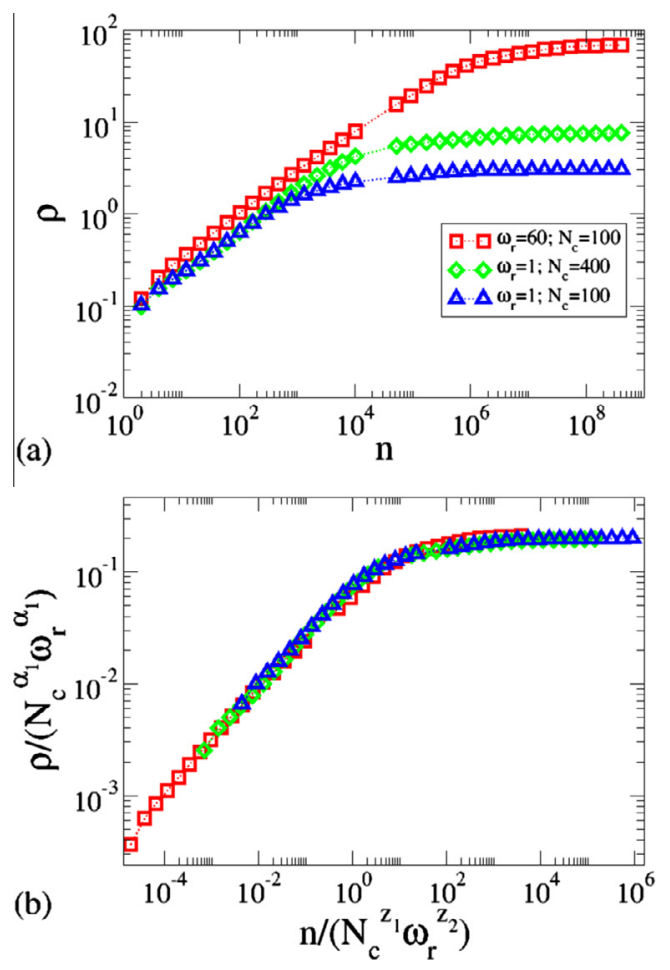

Fig. 9. Plot of: (a) $\rho \times n$ for three different values of $N_{c}$ and $\omega_{r}$; (b) After a rescale of the axis, all curves shown in (a) overlap each other onto a single and universal plot. The parameters used were $r_{1}=r_{2}=1, \delta_{r}=0.5$ and $\delta_{1}=\delta_{2}=0.2$.

(a)
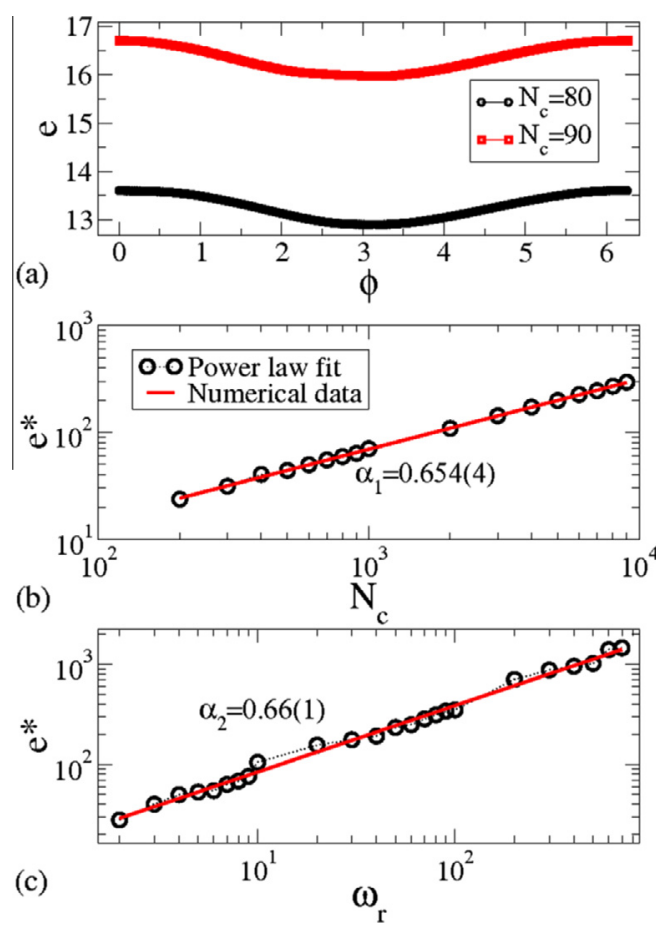

Fig. 10. (a) Position of the lowest invariant KAM curve for two values of $N_{c}$ using $\omega_{r}=1$; (b) $e^{*} \times N_{c}$ for $\omega_{r}=1$; (c) $e^{*} \times \omega_{r}$ for $N_{c}=100$ and using only integer values of $\omega_{r}$. 
ping are observed when they indeed are for $h$ above the islands. This procedure allows us to obtain some properties for transport of particles along the chaotic sea.

Fig. 11(a) shows a plot of a histogram of frequency for the orbits that reach the position $h$ in the phase space at certain $n$. One sees that the histogram exhibits a peak at $n_{p}$ characterizing a most probably $n$ that each particle reaches $h$. Considering that the phase space is mixed and periodic islands are present, the tail observed at long $n$ is an evidence of sticky orbits in the phase space. Some initial conditions can indeed reach $h$ quick, as the short time dynamics indicates $1<n<n_{p}$. They lead the survival probability of particles along the dynamics to be exponential. Trapping near periodic regions however turns the fast exponential survival probability decay into a slower decay that may be either a power law or a stretched exponential [25]. The long tail of the survival probability characterized by a power law was also observed from orbits lying in metastable estate in a double well potential [15]. We are then interested to describe the behavior of $n_{p}$ as a function of the parameters $N_{c}$ and $\omega_{r}$, as shown in Fig. 11(b) and (c). Fitting a power law on the curves shown in Figs. 11(b) and (c) we obtain that the slopes are given by $z_{1}=1.37(4)$ and $z_{2}=1.37(4)$ respectively.

The sticky behavior produces orbits which spend long time dynamics to reach the energy $h$ therefore leading to a probability that some orbits survive long the dynamics. This situation is observed when $h$ is above the first periodic islands. When the dynamics is fully chaotic, the survival orbits decay exponentially in time while the sticky dynamics, produced by orbits visiting very close periodic regions lead to a slower decay (see Ref. [3] for a specific application). The probability that the orbits survive long the dynamics is defined as

$$
P=\frac{1}{N} \sum_{j=1}^{N} N_{\text {surv }}(n),
$$

where the summation is taken along the ensemble of $N$ different initial conditions while $N_{\text {surv }}(n)$ is the number of initial conditions which do not reach $h$ until a time $n$. Fig. 12(a) shows a plot of the survival probability as a function of $n$. The major part of the plot is characterized by an exponential decay with slope $\epsilon$. Plotting $-\epsilon \times N_{c}$ and $-\epsilon \times \omega_{r}$, as shown in Fig. 12(b) and $(\mathrm{c})$, one sees that the slopes are respectively $z_{1}^{\prime} \approx-1.366(9)=-z_{1}$ and $z_{2}^{\prime} \approx-1.36(4)=-z_{2}$. These two exponents, despite the reversed sign, are numerically close to the exponents $z_{1}$ and $z_{2}$ obtained before using scaling approach.

The localization of the invariant KAM curve is not an easy task as it seems to be at first sight. Indeed, the commonly procedure used in the literature to estimate analytically the position of the KAM curves in the phase space is a connection with the standard map [24]. Therefore this procedure assumes that, locally, the dynamics of the system is described by the standard mapping. Given the invariant KAM curve is the lowest one in the energy axis, one may argue that the phase space is separated in two portions: (i) below of the first invariant KAM curve, where global chaos is observed and; (ii) above of the first invariant KAM curve, where local chaos is observed. Therefore there is a transition from local (above the first invari-
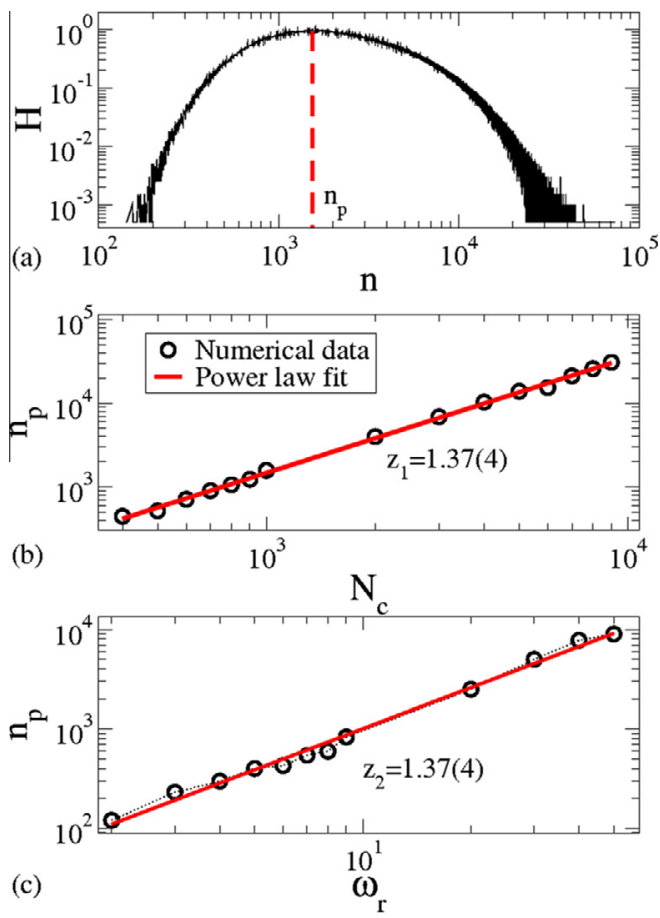

Fig. 11. (a) Plot of the histogram of frequency of orbits reaching the energy $h$ for the parameter $N_{c}=1000$; (b) plot of $n_{p} \times N_{c}$ for $\omega_{r}=1$; (c) plot of $n_{p} \times \omega_{r}$ for $N_{c}=100$. 

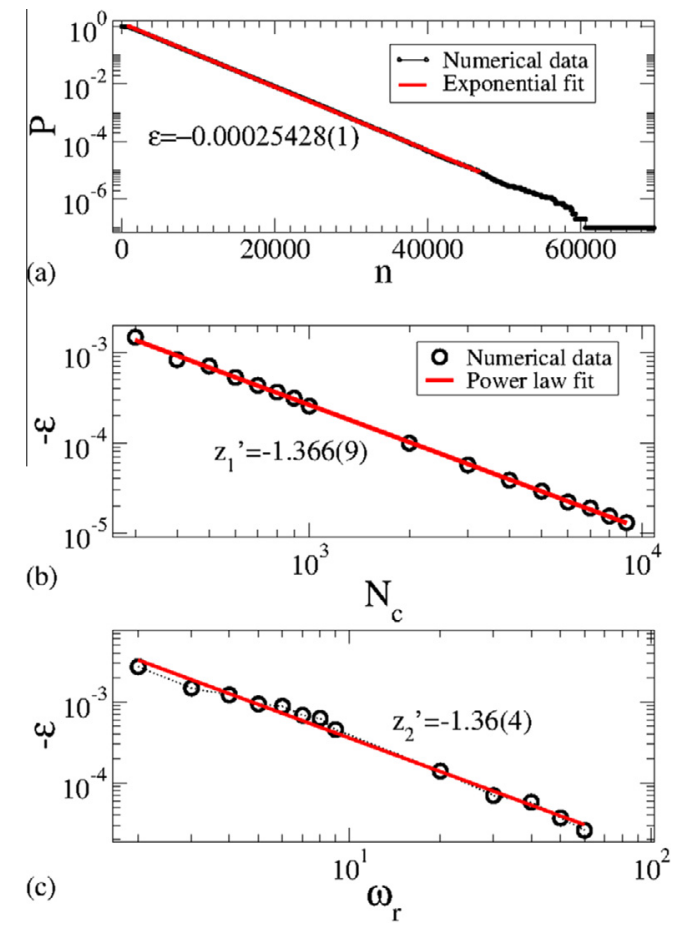

Fig. 12. (a) Plot of the survival probability for $N_{c}=1000$, where the slope of the exponential fit is equal to $\epsilon$; (b) $-\epsilon \times N_{c}$ for $\omega_{r}=1$; (c) $-\epsilon \times \omega_{r}$ for $N_{c}=100$.
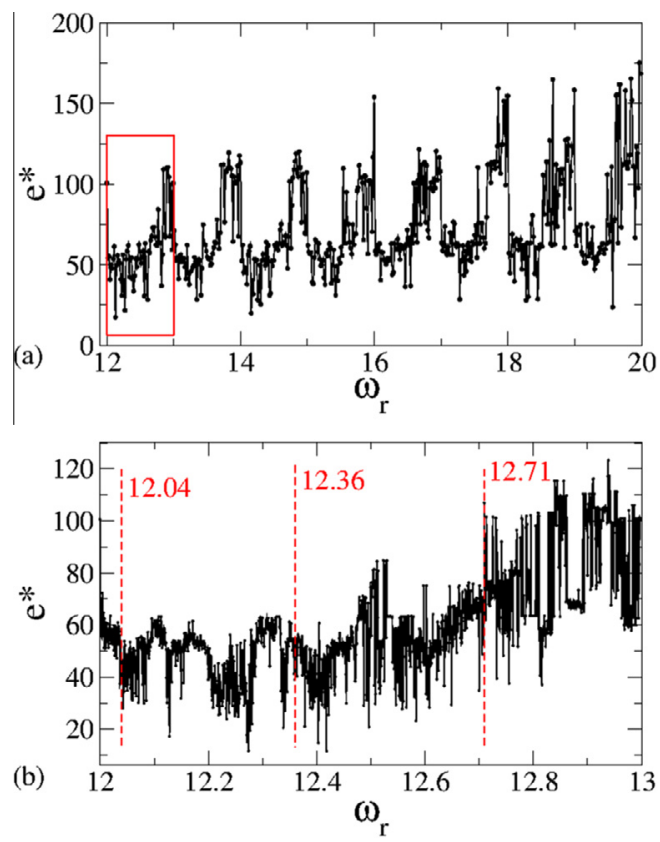

Fig. 13. Magnification of the Fig. 10 (c) showing details about the position of the first invariant spanning curve as function of $\omega_{r}$; (b) Another magnification represented by the red rectangle in the item (a), where the dotted lines represent regions where a specific joining of two fixed points and its vicinity. (For interpretation of the references to color in this figure legend, the reader is referred to the web version of this article.)

ant KAM curve) to global chaos (below the first invariant KAM curve), and in the standard mapping, the critical value of the parameter which controls such transition is around $K_{c} \approx 0.9716 \ldots$.. However this procedure can only be made in a family of mappings whose nonlinearity is the same as that of the standard mapping. In the present case this procedure is impossible. 

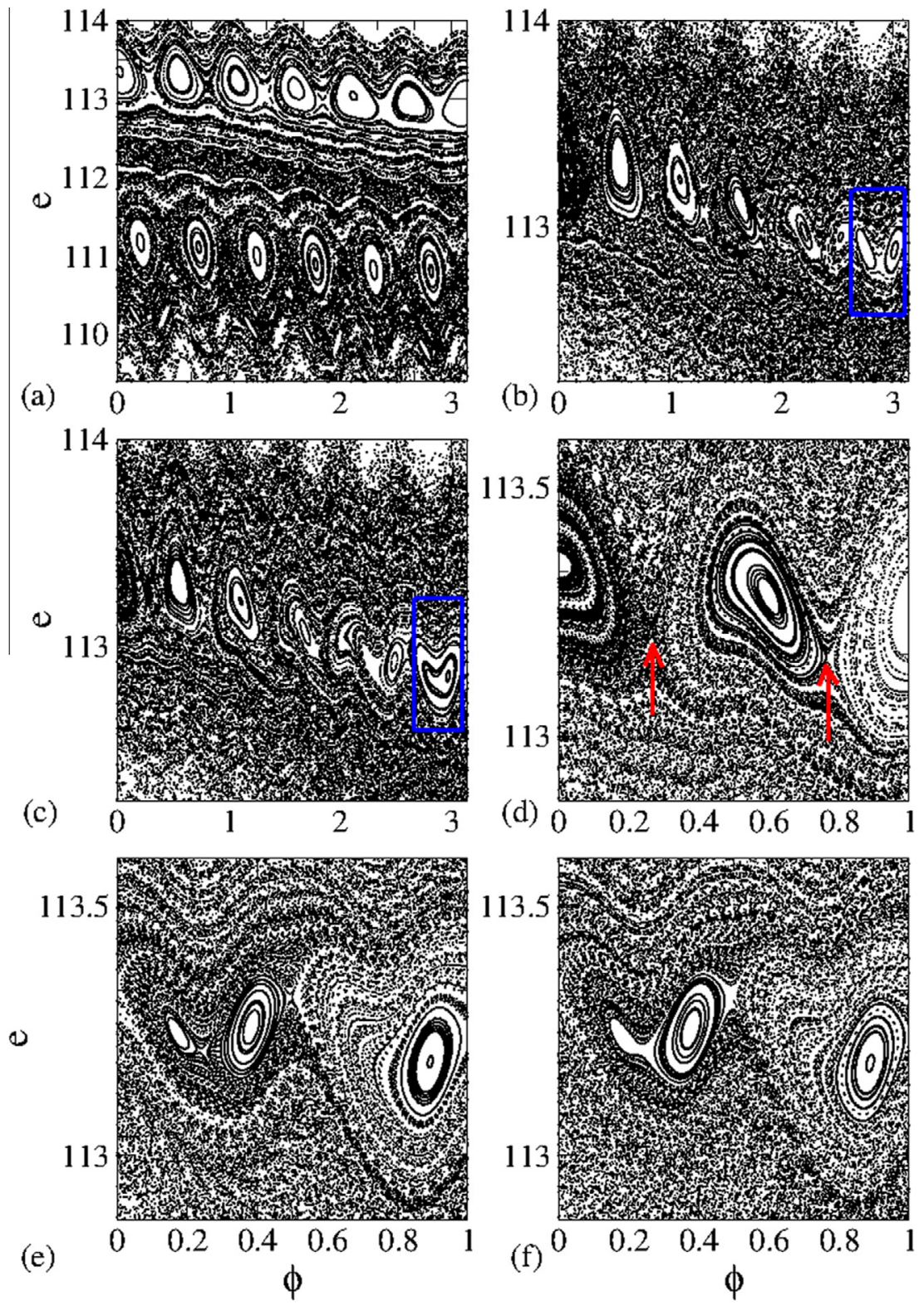

Fig. 14. Phase space for $\omega_{r}$ equal to: (a) 12 ; (b) 12.016 ; (c) 12.02 ; (d) 12.02756 ; (e) 12.039468 ; (f) 12.039884 .

Therefore we recur to numerical simulation as an attempt to estimate the position of such curve. However, the variation of the parameter $\omega_{r}$ in a small range may produce relatively large fluctuations in the position of the lowest invariant KAM curve. To illustrate this, Fig. 13(a) shows a plot of the position of the first invariant KAM curve $e^{*}$ (obtained numerically) as a function of $\omega_{r}$. We see that for $12 \leqslant \omega_{r}<16$ the maximum values of $e^{*}$ fluctuates around 100 and 150 .

Fig. 13(b) shows a magnification of Fig. 13(a) and a complicate behavior of $e^{*}$ is visible in the sense it fluctuates quite a lot. The variation of the parameter $\omega_{r}$ causes modifications in the phase space of the system leading the dynamics to experience trapping in many sticky regions as shown in Fig. 14(a) for $\omega_{r}=12$. For visual purposes, the phase space was limited to $0 \leqslant \phi \leqslant \pi$. As one sees, there are many chains of periodic islands and invariant KAM curves preventing the particle to diffuse in energy. Let us now focus in a more microscopy region, indeed the chain of periodic islands located at $e \cong 113$. As we can see from Fig. 14(a) there is a set of seven large visible islands on the top. If $\omega_{r}$ is changed to 12.016, we note that the first island in the left hand side almost disappear, leading to an appearance of a dark region in the phase space, apparently marked by a sticky region. On the other hand, in the right hand side, there is an apparent period duplication (appearing eight visible islands) and deformation in the shape of the periodic regions as well as its vicinity. We have just highlighted two islands inside the (blue) rectangle just to show that changing a little the parameter $\omega_{r}$ to 12.02 it causes a combination 
of these islands, as we can see in the Fig. 14(c). It happens for other islands too, and many deformations of the periodic islands, apparent duplications and combinations forming new islands are observed. Now changing the parameter $\omega_{r}$ to 12.02756, as shown in Fig. 14(d), one may see structures similar to separatrix, indicated by the red arrows. The last plot shows an enlargement for the region of $\phi \in[0,1]$. Changing yet more the parameter $\omega_{r}$ to 12.039468 (see the Fig. 14(e)) one sees that the first visible periodic island in the left corner is dislocated to the right and an approach to the other island is observed. Increasing $\omega_{r}$ to 12.039884 a joining of the two islands is observed forming a new periodic island. Therefore after increasing a little $\omega_{r}$, the islands come back to the original form as shown in Fig. 14(a). The points where this joining was observed are around $\omega_{r}$ equal to 12.03, 12.36 and 12.71. This behavior was also observed for other ranges of parameters too. Indeed we observed it for $\omega_{r}$ equal to 15.05, 15.386 and 15.722 and around 20.066, 20.40 and 20.734 .

\section{Summary and conclusions}

As a short summary, we described some dynamical properties for chaotic orbits along the phase space for the problem of a classical particle confined inside a box of potential containing a time varying step potential. The equations describing the dynamics were carefully constructed. We obtained that the histogram of frequency for the successive reflections is described by a power law with exponent -3 . This is the same exponent observed for the distribution of traversal time in the problem of a particle moving in a time dependent barrier [21]. The exponent however is not universal given the successive reflections for a beam of light confined in a corrugated waveguide is described with an exponent -3.76 (see Ref. [22]). Using three scaling hypotheses, we obtained scaling exponents and used them to overlap different curves of deviation around the average energy onto a single plot. Such approach was possible due to the existence of a set of invariant KAM curves, preventing the particle to reach unbounded energy. Transport properties along the chaotic sea was studied by seeking the number of iterations needed to reach an energy $h$. The histogram of particles reaching the energy $h$ at a certain $n$ has a peak at $n_{p}$ that is described by a power law. Sticky regions produce trapping and therefore long time dynamics around periodic regions leading orbits to survive long before reach the energy $h$. The survival probability has an exponential decay for low $h$ where periodic regions are absent. However as $h$ rises, a turn from exponential to power law is observed. The slow decay is related to stickiness of orbits trapped around the periodic regions. Similar behavior was observed for the problem of a double well where particles were escaping from one well to the other one due to the effect of noise [15]. The power law fitting of the survival probability was also observed for a double well potential due to orbits lying in metastable state. Therefore the survival probability decays exponentially fast in time for short time and change to slower decay for long time. The slower decay is observed mainly due to trapping in sticky domains. Trapping regions were also observed near the invariant KAM curve as the parameter $\omega_{r}$ is varied. They show to exhibit reorganization of the islands and appearance of seemingly separatrix curves.

\section{Supplemental data}

As a complement of Fig. 13 we can see a supplemental data that include videos in which can be found with this article online or at the following addresses: http://www.youtube.com/watch?v=pQLrxOszedw and http://www.youtube.com/ watch?v=aP3ssNRHAQw. We have considered $\omega_{r} \in[12,13]$ and $\omega_{r} \in[12.026,12.052]$ respectively.

\section{Acknowledgments}

DRC acknowledges Brazilian agency CAPES, CNPq and FAPESP (2010/52709-5 and 2012/18962-0). ILC thanks to CNPq and FAPESP. EDL thanks to CNPq, FUNDUNESP and FAPESP (2012/23688-5, 2008/57528-9 and 2005/56253-8), Brazilian agencies. This research was supported by resources supplied by the Center for Scientific Computing (NCC/GridUNESP) of the São Paulo State University (UNESP).

\section{Appendix A. Supplementary data}

Supplementary data associated with this article can be found, in the online version, at http://dx.doi.org/10.1016/ j.amc.2014.03.021.

\section{References}

[1] M. Leng, C.S. Lent, Recovery of quantized ballistic conductance in a periodically modulated channel, Phys. Rev. Lett. 71 (1993) 137-140.

[2] G.A. Luna-Acosta, G. Orellana Rivadeneyra, A. Mendoza-Galvn, C. Jung, Chaotic classical scattering and dynamics in oscillating 1-D potential wells, Chaos Solitons Fract. 12 (2001) 349-363.

[3] D.R. da Costa, C.P. Dettmann, E.D. Leonel, Escape of particles in a time-dependent potential well, Phys. Rev. E 83 (2011) 066211.

[4] O. Farago, Y. Kantor, Directed chaotic motion in a periodic potential, Physica A 249 (1998) 151-155.

[5] P.S.S. Guimarães et al, Photon-mediated sequential resonant tunneling in intense terahertz electric fields, Phys. Rev. Lett. 70 (1993) $3792-3795$.

[6] D.W. Zhang et al, Josephson dynamics of a spin-orbit-coupled Bose-Einstein condensate in a double-well potential, Phys. Rev. A 85 (2012) 043609.

[7] H. Cao, L.B. Fu, Quantum phase transition and dynamics induced by atom-pair tunnelling of Bose-Einstein condensates in a double-well potential, Eur. Phys. J. D 66 (2012) 97. 
[8] B. Cui, L.C. Wang, X.X. Yi, Time-dependent self-trapping of Bose-Einstein condensates in a double-well potential, Phys. Rev. A 82 (2010) 062105.

[9] M. Bagheri et al, Dynamic manipulation of nanomechanical resonators in the high-amplitude regime and non-volatile mechanical memory operation, Nat. Nanotechnol. 6 (2011) 726-732.

[10] L.F. Buchmann, L. Zhang, A. Chiruvelli, P. Meystre, Macroscopic tunneling of a membrane in an optomechanical double-well potential, Phys. Rev. Lett. 108 (2012) 210403.

[11] L. Chun-Lei, X. Yan, Influence of time-periodic potentials on electronic transport in double-well structure, Chin. Phys. B 19 (2010) 057202.

[12] Q.T. Xie, New quasi-exactly solvable double-well potentials, J. Phys. A: Math. Theor. 45 (2012) 175302.

[13] V.M. Rozenbaum, I.V. Shapochkina, Quasiequilibrium directed hopping in a time-dependent two-well periodic potential, Phys. Rev. E 84 (2011) 051101.

[14] S. Upadhyay, Fokker-Planck equation for time-dependent double-well potentials, J. Phys. A: Math. Gen. 24 (1991) L1293.

[15] B. Dybiec, Escape from the potential well: competition between long jumps and long waiting times, J. Chem. Phys. 133 (2010) 244114.

[16] J.A. de Oliveira, R.A. Bizão, E.D. Leonel, Finding critical exponents for two-dimensional Hamiltonian maps, Phys. Rev. E 81 (2010) 046212.

[17] N.N. Thyagu, N. Gupte, Transport and diffusion in the embedding map, Phys. Rev. E 79 (2009) 066203.

[18] C.P. Dettmann, O. Georgiou, Survival probability for the stadium billiard, Physica D 238 (2009) 2395-2403.

[19] C.P. Dettmann, O. Georgiou, Transmission and reflection in the stadium billiard: time-dependent asymmetric transport, Phys. Rev. E 83 (2011) 036212.

[20] D.E. Sigeti, Exponential decay of power spectra at high frequency and positive Lyapunov exponents, Physica D 82 (1995) 136.

[21] J.L. Mateos, Traversal-time distribution for a classical time-modulated barrier, Phys. Lett. A 256 (1999) 113-121.

[22] M.R. da Silva, D.R. da Costa, E.D. Leonel, Characterization of multiple reflections and phase space properties for a periodically corrugated waveguide, J. Phys. A: Math. Theor. 45 (2012) 265101.

[23] J.P. Eckmann, D. Ruelle, Ergodic theory of chaos and strange attractors, Rev. Mod. Phys. 57 (1985) 617.

[24] E.D. Leonel, J.A. de Oliveira, F. Saif, Critical exponents for a transition from integrability to non-integrability via localization of invariant tori in the Hamiltonian system, J. Phys. A: Math. Theor. 44 (2011) 302001.

[25] C.P. Dettmann, E.D. Leonel, Escape and transport for an open bouncer: stretched exponential decays, Physica D 241 (2012) 403. 